\title{
Cost-efficient Distributed Optimization In Machine Learning Over Wireless Networks
}

\author{
Afsaneh Mahmoudi, Hossein S. Ghadikolaei, and Carlo Fischione \\ Network and Systems Engineering, Electrical Engineering and Computer Science \\ KTH Royal Institute of Technology, Stockholm, Sweden \\ emails: \{afmb, hshokri, carlofi $\} @$ kth.se
}

\begin{abstract}
This paper addresses the problem of distributed training of a machine learning model over the nodes of a wireless communication network. Existing distributed training methods are not explicitly designed for these networks, which usually have physical limitations on bandwidth, delay, or computation, thus hindering or even blocking the training tasks. To address such a problem, we consider a general class of algorithms where the training is performed by iterative distributed computations across the nodes. We assume that the nodes have some background traffic and communicate using the slotted-ALOHA protocol. We propose an iteration-termination criterion to investigate the trade-off between achievable training performance and the overall cost of running the algorithms. We show that, given a total running budget, the training performance becomes worse as either the background communication traffic or the dimension of the training problem increases. We conclude that a codesign of distributed optimization algorithms and communication protocols is essential for the success of machine learning over wireless networks and edge computing.
\end{abstract}

Index Terms-Distributed optimization, efficient algorithm, latency, convergence, machine learning.

\section{INTRODUCTION}

The recent success of artificial intelligence (AI) and largescale machine learning (ML), are largely based on the advancements of distributed optimization algorithms [1]. The main objective of such algorithms is better training/test performance. However, the distributed optimizations do not include by design the costs of running the solution algorithms. The state-of-the-art of such algorithms requires powerful computing platforms with vast amounts of computational and communication resources. Although such resources are available in modern data centers, there is an emerging need to extend machine learning tasks where such powerful computing platforms are not available due to communication and energy resource constraints. Use cases include machine leaning over Internet-of-Things, edge computing, vehicular networks, mobile communications, or general networks with background traffic [2], [3].

In this paper, we address the problem of distributed optimization for ML over a wireless communication network, where the computations and communications must necessarily be efficient. We call such a problem "ML over networks" (MLoNs). Specifically, our MLoN is defined as follows: We have a star network topology where a master node owns the ML model and a set of distributed worker nodes $[N]$ owns the data. The ML problem consists in optimizing a finite sum of $N$ differentiable functions $f_{i}, i \in[N]$, which take inputs from $\mathbb{R}^{d}$ for some positive $d$ and give their outputs in $\mathbb{R}$, i.e., $\left\{f_{i}: \mathbb{R}^{d} \mapsto \mathbb{R}\right\}_{i \in[N]}$ with corresponding gradients $\left\{\nabla f_{i}: \mathbb{R}^{d} \mapsto \mathbb{R}^{d}\right\}_{i \in[N]}$. This problem is predominant in ML training [4]. The common solution to such problems involves an iterative procedure wherein at iteration $k$, worker nodes have to find the gradients $\left\{\nabla f_{i}\left(\boldsymbol{w}_{k}\right)\right\}_{i}$ at parameter $\boldsymbol{w}_{k}$, send them back to a central controller (master node). Then, the master node updates the model parameters to $\boldsymbol{w}_{k+1}$ and broadcasts it to all the nodes so they can start the next iteration [5]. However, every iteration of these algorithms imposes some costs (e.g., computation, privacy, latency, or energy). Although distributed optimization algorithms alleviate computation and privacy by parallel computations at worker nodes using their local private data [5], [6], they introduce a communication cost: parameter and gradient vectors should be exchanged among the master and the worker nodes to run a new iteration. As we show throughout this paper, the communication cost can be orders of magnitude larger than the computational costs, thus making the iterative procedure much inefficient.

There have been numerous studies on communicationefficient optimization and distributed ML. These studies can be grouped into three main approaches:

- Regret minimization provides a framework for characterizing cost-efficient iterative algorithms, when the cost is defined as the optimality penalty, $\sum_{i \in[N]} f_{i}\left(\boldsymbol{w}_{k}\right)-$ $\sum_{i \in[N]} f_{i}\left(\boldsymbol{w}^{\star}\right)$, where $\boldsymbol{w}^{\star}$ is the optimal solution [7]. An important weakness of regret minimization framework is the decreasing additional penalty over time. Namely, as we get closer to the minimizer, $\sum_{i \in[N]} f_{i}\left(\boldsymbol{w}_{k}\right)-$ $\sum_{i \in[N]} f_{i}\left(\boldsymbol{w}^{\star}\right)$ reduces. However, in the presence of a real communication network, the iteration costs may be actually increasing (not decreasing) function of the iterations. Consequently, the regret minimization framework may lead to a huge number of iterations and thus of communications, as we shall see in Section IV-A.

- Compression is an approach to reduce the amount of information exchange (bits) among distributed nodes, and thereby save communication resources [8], [9]. This can be realized through the quantization of the parameter and gradient vectors by fewer bits or sending only the most important values (the ones with higher magnitudes). 
- Model averaging describes an alternative approach wherein worker nodes update their local models, and these models are averaged across nodes after some iterations. The nodes then start their local updates using this new global parameter and continue this process until convergence [10]. Reference [10] showed that these approaches can substantially reduce the number of communication rounds needed to solve an optimization problem.

Unfortunately, the aforementioned approaches fail to capture the actual cost of running a distributed optimization algorithm and its adaptation to the underlying communication protocol. The same compression or model averaging algorithm may correspond to completely different values for latency or energy consumption in various communication settings. The main problem is that the three existing approaches mentioned above assume that the complexity of a distributed MLoN is given by the number of bits per communication round, or the number of communication rounds. However, they neglect other more important costs involved in MLoNs: overall latency and energy consumption for solving a ML problem. These factors become of paramount importance when we implement machine learning on bandwidth or battery-limited wireless networks, where latency (e.g., in remote surgery application) and energy consumption (e.g., in low power Internet-ofThings) may render the ultimate solution and consequently the distributed algorithm useless. This paper address this important research gap.

In this paper, we characterize the overall latency cost of solving an MLoN problem where the worker nodes have some background traffics and follow a slotted-ALOHA medium access control protocol for uplink. The simple protocol requires no coordination among the worker nodes and provides a reasonable lower bound on the convergence rate of the distributed optimization algorithm [11]. Moreover, in the presence of directional communication, slotted-ALOHA provides a better latency performance than many other contentionbased medium access control algorithms [12]. We propose to optimize the trade-off between achievable objective value and the overall cost of running the distributed ML algorithm. We then propose a non-causal solution algorithm to such an optimization problem as a means to characterize the minimum. Afterward, we develop a simple causal solution algorithm that can find a point very close to the non-causal minimum. Our extensive results show that, given a total budget to run the iterations, the ML training performance gets worse as either the background communication traffic or the dimension of the optimization problem increases. We observe similar patterns in both batch and mini-batch updates of our proposed algorithm. We conclude that a co-design of distributed optimization algorithms and communication protocols are essential for the success of MLoN, including its applications to edge computing, Internet-of-Things, or intelligent transportation systems.

The rest of this paper is organized as follows. Section II and Section III describe the general system model and some following results. In Section IV, we analyze the proposed batch and mini-batch updates with some numerical results and concluding remarks are presented in Section V.

Notation: Normal font $\boldsymbol{w}$, bold font small-case $\boldsymbol{w}$, boldfont capital letter $\boldsymbol{W}$, and calligraphic font $\mathcal{W}$ denote scalar, vector, matrix, and set, respectively. We define the index set $[N]=\{1,2, \ldots, N\}$ for any integer $N$. We denote by $\|\cdot\|_{p}$ the $l_{p}$ norm for $p \in \mathbb{N} \cup\{\infty\}$, by $\|\cdot\|$ the $l_{2}$ norm, by $[\boldsymbol{w}]_{i}$ entry $i$ of vector $\boldsymbol{w}$, and by $\boldsymbol{w}^{\mathrm{T}}$ the transpose of $\boldsymbol{w}$.

\section{SYSTEM MODEL}

Consider a network of $M$ computational (worker) nodes that cooperatively solve a distributed computational problem involving an objective function $f(\boldsymbol{w})$. Let tuple $\left(\boldsymbol{x}_{i j}, y_{i j}\right)$ denote data sample $i$ of worker node $j$ and $\boldsymbol{w}$ denote the model parameter at the master node, with dimension $d$. Assuming $\sum_{j \in[M]} N_{j}=N$, our optimization problem is

$$
\boldsymbol{w}^{\star} \in \min _{\boldsymbol{w} \in \mathbb{R}^{d}} f(\boldsymbol{w})=\frac{1}{M} \sum_{j \in[M]} \underbrace{\frac{1}{N_{j}} \sum_{i \in\left[N_{j}\right]} f\left(\boldsymbol{w} ; \boldsymbol{x}_{i j}, y_{i j}\right)}_{:=f_{j}(\boldsymbol{w})} .
$$

We assume that our objective function $f$, is convex and $L$ smooth and we use a gradient descent solver for obtaining the optimal solution of $f$. We need these assumptions for the analysis, though our approach is applicable to multiconvex [13] structures (such as a deep neural network training optimization problem) after some minor modifications.

\section{A. Gradient Descent}

Gradient descent is a prominent family of algorithms to solve (1) by distributed computations among worker nodes. The master node sends $\boldsymbol{w}_{k}$ to the worker nodes at iteration $k$ and then each worker node $j$ computes in parallel its gradient term $\nabla_{k}^{j}:=\left\{\sum_{i \in\left[N_{j}\right]} \nabla_{w} f\left(\boldsymbol{w}_{k} ; \boldsymbol{x}_{i j}, y_{i j}\right) / N_{j}\right\}$ over its private dataset of $N_{j}$ samples. Afterward, they send the gradient vectors to the master node, who updates the parameter according to

$$
\boldsymbol{w}_{k+1}=\boldsymbol{w}_{k}-\alpha_{k} \frac{\sum_{j \in[M]} \nabla_{k}^{j}}{M},
$$

for a sequence of positive step-sizes $\left(\alpha_{k}\right)_{k=0,1, \ldots, K}$. The master node runs these iterations until a convergence criteria is met. These steps are summarized in the well-known Algorithm 1. Let $\bar{k}$ shows the iteration at which the stopping criteria is met for the first time, namely

$$
\bar{k}=\min \left\{k \mid\left\|\frac{1}{M} \sum_{j \in[M]} \nabla_{k}^{j}\right\|<\varepsilon_{k}\right\},
$$

where $\varepsilon_{k}>0$ is the decision threshold for terminating the algorithm at iteration $k$. Let $c_{k}$ denote the cost of iteration $k$. Running Algorithm 1 costs $\sum_{k=1}^{\bar{k}} c_{k}$.

We assume that the sequence $\left(c_{k}\right)_{k=1,2, \ldots}$ is independent of objective function $f$, the decision threshold $\left(\varepsilon_{k}\right)$, and parameter $\left(\boldsymbol{w}_{k}\right)$. A mission-critical use case has, for example, $c_{k} \rightarrow \infty$ when $k>K$ for some positive integer $K$ (decision deadline). This means that after a decision deadline, the cost 
Algorithm 1: Generic gradient descent algorithm model.

1: Inputs: $\boldsymbol{w}_{0}, f,\left(\boldsymbol{x}_{i j}, y_{i j}\right)_{i, j}, \alpha_{k}, K, M,\left\{N_{j}\right\}_{j \in[M]}$

: for $k=1,2, \ldots, K$ do

Each node $j \in[M]$ does in parallel

Obtain $\nabla_{k}^{j}:=\left\{\sum_{i \in\left[N_{j}\right]} \nabla_{w} f\left(\boldsymbol{w}_{k} ; \boldsymbol{x}_{i j}, y_{i j}\right) / N_{j}\right\}$

Send $\nabla_{k}^{j}$ to the master node

6: $\quad$ Master node collects $\left\{\nabla_{k}^{j}\right\}_{j}$ and runs

$$
\boldsymbol{w}_{k+1} \leftarrow \boldsymbol{w}_{k}-\alpha_{k} \frac{\sum_{j \in[M]} \nabla_{k}^{j}}{M}
$$

7: $\quad$ Master node broadcasts $\boldsymbol{w}_{k+1}$ to the worker nodes

8: end for

9: Return $\boldsymbol{w}_{K+1}$

to further run the algorithm diverges to infinity. In the next sections, we characterize $\left(c_{k}\right)_{k}$ for two specific use cases when we consider the latency cost of every iteration.

\section{B. Problem Formulation}

We wish to solve problem (1) with the gradient descent method, but where we explicitly consider the cost associated with the iterations. Specifically, we define the general cost as a convex combination of the iteration-cost function $\sum_{k=1}^{\bar{k}} c_{k}$ and the loss function $f\left(\boldsymbol{w}_{\bar{k}}\right)$. Note that such a cost is general in the sense that, depending on the values of $c_{k}$, it can naturally model many cases including constant charge per computation and mission-critical application, among others. Assuming a sufficiently large integer $K$, we have:

$$
\underset{\varepsilon_{1}, \varepsilon_{2}, \ldots, \varepsilon_{K}}{\operatorname{minimize}} \beta \sum_{k=1}^{K} c_{k} \mathbf{1}_{k \leq \bar{k}}+(1-\beta) f\left(\boldsymbol{w}_{\bar{k}}\right),
$$

where $\mathbf{1}_{x}$ is an indicator function taking 1 iff $x$ is true, and $\bar{k}$ shows the index at which we stop the iterations of the distributed gradient algorithm.

Optimization problem (3) addresses the tradeoff between the iteration-cost function $\sum_{k=1}^{k} c_{k}$ and the loss function $f\left(\boldsymbol{w}_{\bar{k}}\right)$. First, the "descent" property of Algorithm 1 implies that $\left\|\sum_{j \in[M]} \nabla_{k}^{j} / M\right\|$ is a non-increasing (usually decreasing) function of $k$. Consequently, equation (5) yields that $\bar{k}$ is a non-increasing function of $\varepsilon_{k}$. On one hand, increasing $\bar{k}$ (equivalently reducing $\varepsilon_{k}$ ) requires additional transmissions of parameter and gradient and vectors among the worker nodes and the master node, and consequently an increase of the cost of running gradient descent. On the other hand, the gradient descent iterations ensure that $f\left(\boldsymbol{w}_{\bar{k}}\right)$ decreases with $\bar{k}$. Optimization problem (1) addressed this tradeoff by finding the optimal sequence of termination thresholds $\left(\varepsilon_{k}\right)_{k}$. Once the optimal sequence is known after solving optimization problem (3), we can achieve an efficient distributed gradient descent algorithm to solve (1). We give a concrete definition for this efficiency in the following subsection.

\section{Slotted-ALOHA example}

Although our framework is applicable to a general cost model, we focus on the end-to-end latency as the iterationcost function $\sum_{k} c_{k}$. To have a concrete model of latency, we consider the following wireless communication scenario. We consider a broadcast channel in the downlink (from the master node to the worker nodes) and a contention-based multiple access channel in the uplink (from the worker nodes to the master node). We assume that the uplink transmissions are regulated by slotted-ALOHA protocol [11]. Every node has an infinite buffer to keep their information packets, including gradient vectors, and send them by the first-in-first-out policy. Upon receiving $\boldsymbol{w}_{k}$, each worker node $j(\in[M])$ computes its average gradient term $\nabla_{j}$ and puts it in its transmission queue as shown in Fig. 1. Once a packet is at the queue head, it follows slotted-ALOHA with a binary exponential backoff retransmission policy [14]. In particular, the transmission starts only at the beginning of the slots and upon every collision, one retransmission is scheduled after a random backoff delay taken uniformly at random from window $[1, b]$ for some integer $b$ that is doubled by every collision event.

Running every iteration of Algorithm 1 entails four latency components:

$\ell_{1}$ : latency in broadcasting parameters by master node,

$\ell_{2}$ : latency in computing $\nabla_{k}^{j}$ for every worker node $j$,

$\ell_{3}$ : latency in sending $\nabla_{k}^{j}$ to master node in a multiple access channel, and

$\ell_{4}$ : latency in updating parameters at the master node.

For $\ell_{1}$, we consider a broadcast channel with data rate $C$ bits/sec and parameter size of $b$ bits (which includes the payload and headers), leading to a constant latency of $\ell_{1}=b / C$ seconds. It is natural to assume that $\ell_{2}$ and $\ell_{4}$ are constant and given. These values can be easily estimated by the clock speed of the processors and the workload of computing gradients at the worker nodes or updating parameters at the master node [15]. The third term, $\ell_{3}$, is usually the largest because it is determined by the channel capacity, resource allocation policy, and the network traffic. In the next sections, we characterize this term for two cases:

- Batch update, where the master node updates $\boldsymbol{w}_{k}$ only after receiving all the gradients $\left\{\nabla_{k}^{j}\right\}_{j \in[M]}$; and

- Mini-batch update, where the master node receives gradients that arrive within window of $T_{s}$ time slots and updates $\boldsymbol{w}_{k}$ afterward with the received gradients.

The batch update is normal gradient descent, and the minibatch update is similar to a mini-batch gradient descent where a worker node $j$ at iteration $k$ computes the gradient $\nabla_{k}^{j}$ with a (usually randomly selected) subset of its private dataset [4]. In many cases with big datasets, this sub-sampling process substantially reduces computational complexity. The main difference of this approach to our mini-batch gradient version is that the wireless communication network is the main reason for updating $\boldsymbol{w}_{k}$ without some gradient terms. In that case, all the computations done for some $\nabla_{j}^{k}$ may be a waste if a poor communication channel or heavy background traffic of node $j$ prohibits the master node to receive $\nabla_{j}^{k}$ within $T_{s}$ slots. In Section IV-B, we characterize the impact of $T_{s}$ and the background traffic on the convergence performance of our solution algorithms to (3). 


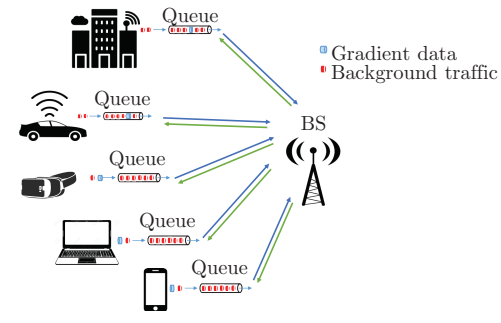

Fig. 1: General architecture of Machine Learning over Networks (MLoNs) problem. The BS is the master node and the various terminals are the worker nodes. They all wish to solve problem (1). The queues at the worker nodes have background traffics (red items) and gradient vectors (blue items), required the distributed gradient descent algorithm using our proposed thresholds obtained by (6).

\section{SOlution Algorithms}

In this section, we derive some useful properties of optimization problem (3). Then, based on those properties, we propose a non-causal and a causal solution algorithms for this optimization problem.

\section{A. Results}

In this part, we aim to discuss about some technically useful results obtained from (3). First we consider the following assumption:

Assumption 1. The iteration-cost function $\sum_{k=1}^{\bar{k}} c_{k}$ is a discrete convex function of $\bar{k}$.

For notation simplicity, define $C_{\bar{k}}:=\sum_{k=1}^{\bar{k}} c_{k}$. Assumption 1 implies that the sequence $\left(C_{\bar{k}}\right)_{\bar{k}}$ satisfies $2 C_{\bar{k}} \leq$ $C_{\bar{k}-1}+C_{\bar{k}+1}$ for all $\bar{k} \in \mathbb{N}$ [16, Definition 1]. Defining $\Delta C_{\bar{k}}=C_{\bar{k}}-C_{\bar{k}+1}$ and $\Delta^{2} C_{\bar{k}}=\Delta C_{\bar{k}}-\Delta C_{\bar{k}+1}$, Assumption 1 implies $\Delta^{2} C_{\bar{k}} \geq 0$ for all $\bar{k} \in \mathbb{N}$. Now, we can pose the following proposition.

Proposition 1. Given a descent-class of algorithms, optimization problem (3) is identical to

$$
\underset{\varepsilon}{\operatorname{minimize}} \beta \sum_{k=1}^{\infty} c_{k} \mathbf{1}_{k \leq \bar{k}}+(1-\beta) f\left(\boldsymbol{w}_{\bar{k}}\right),
$$

where

$$
\bar{k}(\varepsilon):=\min \left\{k \mid\left\|\frac{1}{M} \sum_{j \in[M]} \nabla_{k}^{j}\right\|<\varepsilon\right\} .
$$

Proposition 1 implies that the termination time and the objective value of (3) when utilizing Algorithm 1 are the same for both constant optimal $\varepsilon$ and optimal sequence $\left(\varepsilon_{k}\right)_{k}$. Therefore, we focus only on (4) with the purpose of deriving the stopping time of Algorithm 1.

Lemma 1. Consider $\varepsilon^{\star}$ as a minimizer of optimization problem (4). Let $k^{\star}$ be the solution to the following optimization problem by defining $G(\bar{k}(\varepsilon)):=\beta \sum_{k=1}^{\infty} c_{k} \mathbf{1}_{k \leq \bar{k}}+(1-$ $\beta) f\left(\boldsymbol{w}_{\bar{k}}\right)$.

$$
k^{\star} \in \underset{\bar{k} \in \mathbb{N}}{\arg \min } G(\bar{k}(\varepsilon))
$$

Then $G\left(\bar{k}\left(\varepsilon^{\star}\right)\right)=G\left(k^{\star}\right)$, namely the solution to (6) gives the optimal number of iterations that solves (5).

Proof: Define the pre-image of $\bar{k}(\varepsilon)$ at any integer $k_{0}$ as $\mathcal{E}\left(k_{0}\right):=\left\{\varepsilon \mid \bar{k}(\varepsilon)=k_{0}\right\}$. Now, let $\varepsilon^{\star}$ be a solution to optimization problem (4). By definition, $\bar{k}\left(\varepsilon^{\star}\right)$ minimizes $G$, and therefore $G\left(\bar{k}\left(\varepsilon^{\star}\right)\right) \leq G\left(k^{\star}\right)$. Now, if $\bar{k}\left(\varepsilon^{\star}\right) \neq k^{\star}$ and that $G\left(\bar{k}\left(\varepsilon^{\star}\right)\right) \neq G\left(k^{\star}\right)$, there exists a $k^{\star}$ from (6) that achieves a smaller $G$ which cannot be true due to the optimality of $\varepsilon^{\star}$. Conversely, if $k^{\star}$ is a solution to (6), $G\left(k^{\star}\right) \leq G(\bar{k}(\varepsilon))$ for any $\varepsilon$. Therefore, any $\varepsilon \in \mathcal{E}\left(k^{\star}\right)$ is a solution to optimization problem (4), leading to $G\left(\bar{k}\left(\varepsilon^{\star}\right)\right)=G\left(k^{\star}\right)$.

Lemma 1 indicates that instead of optimizing over $\varepsilon$, we can directly find the optimal stopping iteration by solving optimization problem (5) and achieve the same objective value of (5) by solving optimization problem (6). In the following, we focus on optimization problem (6). Now, we consider two illustrative extreme examples in which there is no tradeoff between two terms of the objective function of (6). The purpose of these examples is to show the importance of costaware distributed optimization in MLoN.

Example 1. Consider $\beta=0$ and $\beta=1$ special cases:

- When $\beta=0$, optimization problem (6) reduces to

$$
k^{\star} \in \underset{\bar{k} \in \mathbb{N}}{\arg \min } f\left(\boldsymbol{w}_{\bar{k}}\right),
$$

in which the iteration cost $c_{k}$ does not play any role in optimization problem, and consequently $k^{\star} \rightarrow \infty$ would be the solution of (6). In other words, due to lack of any penalty in running iterations and convexity of the objective function, the algorithm iterates until $f\left(\boldsymbol{w}_{k}\right) \rightarrow$ $f\left(\boldsymbol{w}^{\star}\right)=f^{\star}$. However, finding this solution may entail a very large iteration cost, ignored in (7). It follows for every $\varepsilon \geq 0$ that $G\left(\varepsilon^{\star} ; \beta=0, \boldsymbol{w}_{K}, c_{k}\right) \leq G(\varepsilon ; \beta=$ $\left.0, \boldsymbol{w}_{K}, c_{k}\right)$.

- When $\beta=1$, optimization problem (6) reduces to

$$
k^{\star} \in \underset{\bar{k} \in \mathbb{N}}{\arg \min } \sum_{i=1}^{\infty} c_{k} \mathbf{1}_{k \leq \bar{k}},
$$

in which the objective function $f\left(\boldsymbol{w}_{k}\right)$ of (1) plays no role in optimization problem (6). Consequently, (8) minimizes only the iterating cost, with a trivial solution of $k^{\star}=$ 0 (stop without any iteration) due to the positively of sequence $\left(c_{k}>0\right)_{k}$.

The examples above teach us that we need to co-design communication protocol (to reduce $\sum_{k} c_{k}$ ) and the optimization algorithm that solves the machine learning problem (to reduce $f$ or stop iterations at the right time without incurring in extra costs). Now we can turn to showing a useful property of optimization problem (6).

Lemma 2. Optimization problem (6) is discrete convex.

Proof: It follows from the definition of discrete convexity [17] and that both terms of the objective function are discrete convex. We use this convexity property in the next subsection to solve (6). 
Lemma 2 plays an important role in order to develop the solution algorithms we present in the next part.

\section{B. Non-causal and Causal Solution Algorithms}

Here, using the results of Subsection III-A, we propose two algorithms to solve optimization problem (6).

1) Non-causal Setting: One of the simplest approaches to solve (6) is an exhaustive search over the discrete set $[0, K]$. To this end, we should have in advance, namely at time $k=0$, the sequence of $\left(f\left(\boldsymbol{w}_{k}\right)\right)_{k}$ and $\left(c_{k}\right)_{k}$ for all $k \in[0, K]$. Although $\left(c_{k}\right)_{k}$ may be known a priory in some use cases, the sequence of parameters $\left(\boldsymbol{w}_{k}\right)_{k}$ and consequently the sequence of $\left(f\left(\boldsymbol{w}_{k}\right)\right)_{k}$ are not available in advance. Our non-causal setting assumes that all these values are available at $k=0$ so it finds $k^{\star}$ of optimization problem (6). Clearly, it is an impractical approach, because obtaining the values of $\left(f\left(\boldsymbol{w}_{k}\right)\right)_{k}$ requires following each iteration $k$ in the gradient descent algorithm while we are supposed to find the optimal termination iteration. However, it gives the true minimizer $k^{\star}$, which we can use later in Section IV to assess the performance of the following causal solution algorithm.

2) Causal Setting: since we cannot construct the non-causal setting to solve (6) in real world, it is critical to develop a practical causal way for solving this important optimization problem. Consider $G(\bar{k}):=\beta \sum_{k=1}^{\bar{k}} c_{k}+(1-\beta) f\left(\boldsymbol{w}_{\bar{k}}\right)$ as the cost function at iteration $\bar{k} \leq k^{\star}$. Since $G(\bar{k})$ is a convex combination of the loss function $f$ and the iterationcost function $\sum_{k} c_{k}$, it is a discrete convex function; see Lemma 2. Therefore, the optimal point is achievable by tracking the sign of derivative of $G(\bar{k})$, which corresponds to the sign of difference of two consecutive $G$. In particular, in the causal setting, we terminate the gradient descent iterations once $G(\bar{k}+1) \geq G(\bar{k})$. Algorithms 2 and 3 illustrate batch and mini-batch implementations of the distributed gradient descent with our causal termination approach. Notice that the implicit sub-sampling (from set $\left\{\nabla_{k}^{j}\right\}_{j}$ ) in the mini-batch updates, which can be interpreted as the stochastic noise [4] leads to approximations of $f\left(\boldsymbol{w}_{k}\right)$, denoted by $\hat{f}\left(\boldsymbol{w}_{k}\right)$, and therefore approximation of cost function, denoted by $\hat{G}(\bar{k})$. This stochastic noise may lead to an improper early stop from $\hat{G}(\bar{k}+1) \geq \hat{G}(\bar{k})$. To address this, Algorithm 3 may wait for some more time slots to get more gradients and have an update with a smaller gradient noise. Once the objective is not reduced for $n_{t}$ consecutive iterations, we stop the mini-batch updates.

Proposition 2. Let $k^{\star}$ and $k_{c}^{\star}$ denote the solutions to optimization problem (6), obtained in the non-causal and causal settings, respectively. We have

$$
\begin{aligned}
k^{\star} \leq k_{c}^{\star} & \leq k^{\star}+1, \\
f\left(k_{c}^{\star}\right) & \leq f\left(k^{\star}\right), \text { and } \\
G\left(\boldsymbol{w}_{k_{c}^{\star}}\right) & \geq G\left(\boldsymbol{w}_{k^{\star}}\right) .
\end{aligned}
$$

Proof: In the beginning, the solver sets $\varepsilon=+\infty$ in order to ensure running of the iterations. Inequality $G(\bar{k}+1)<G(\bar{k})$ implies that the objective function is still in the decreasing
Algorithm 2: Batch update with causal setting.

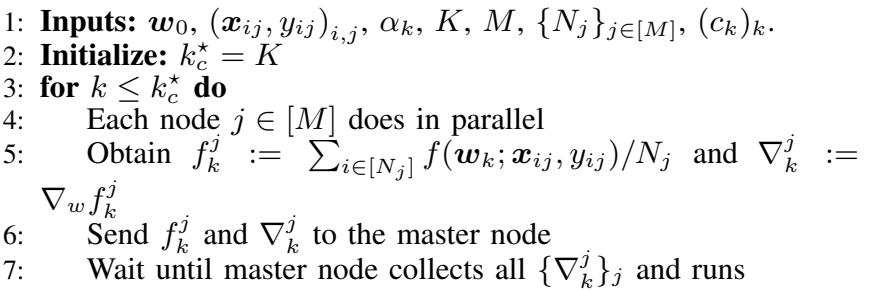

$$
\boldsymbol{w}_{k+1} \leftarrow \boldsymbol{w}_{k}-\alpha_{k} \frac{\sum_{j \in[M]} \nabla_{k}^{j}}{M}
$$
$\begin{array}{ll}\text { 8: } & \text { Calculate } f\left(\boldsymbol{w}_{k}\right):=\sum_{j \in[M]} f_{k}^{j} /|M| \text { and } G(k) \\ \text { 9: } & \text { if } G(k)<G(k-1) \text { then }\end{array}$

11: else

Master node broadcasts $\boldsymbol{w}_{k+1}$ to the worker nodes

12: $\quad$ Set $k_{c}^{\star}=k$, Break and go to line 15

13: $\quad$ end if

14: $\quad$ Set $k \leftarrow k+1$

15: end for

16: Return $\boldsymbol{w}_{k_{c}^{\star}}, k_{c}^{\star}, G$

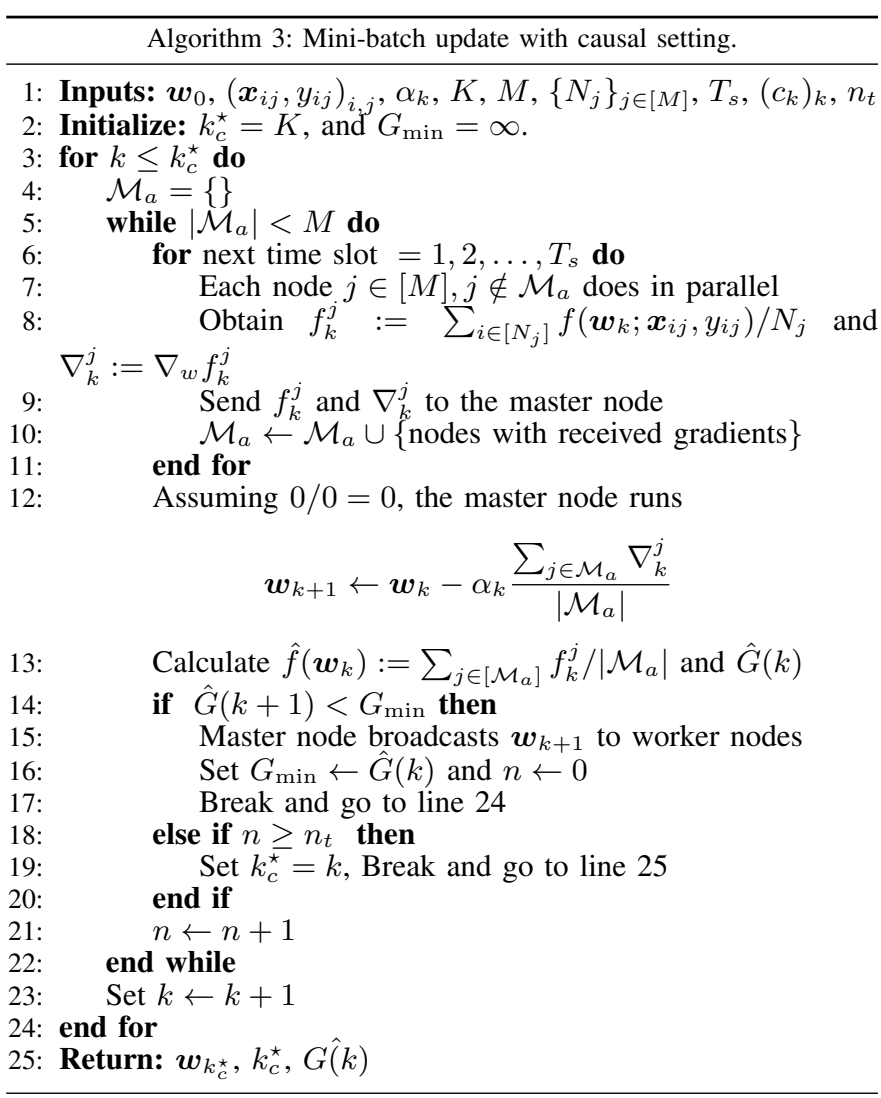

region and we have not found the minimum yet. As soon as the sign changes, the algorithm would terminate by setting the value of $\varepsilon=0$, set the current $k$ as $k_{c}^{\star}$, and then solver reports the corresponding values for $G\left(k_{c}^{\star}\right)$ and $f\left(\boldsymbol{w}_{k_{c}^{\star}}\right)$. Clearly, the penalty is up to one additional iteration when we observe increase in $G$ and terminate the process, leading to inequality (9a). Given (9a), the discrete convexity of the optimization problem (6) and the "descent property" of the 
gradient descent algorithm [4] yields (9b). Inequality (9c) is a direct consequence of the definition of $k^{\star}$ in (6).

Proposition 2, as a central result of our paper, shows that we can develop a simple yet close-to-optimal algorithm for optimization problem (6), and therefore of optimization problem (4) thanks to Lemma 1. In other words, it takes just one iteration more to the causal setting compared to the noncausal.

\section{NuMERICAL RESUltS}

In this section, we illustrate our results from the previous sections and we numerically characterize the latency (cost) of running distributed optimization to solve an ML problem over a wireless network. We use a network simulation to implement slotted-ALOHA with binary exponential backoff and $M$ worker nodes in the network. We consider a capacity of one packet per slot and a slot duration of $1 \mu$ s and assume that every worker node generates a new background traffic packet at every time slot with probability $\frac{p_{b}}{M}$. A higher $p_{b}$ values correspond to intensive traffics, like video streaming, while a lower $p_{b}$ corresponds to lighter traffics, like browsing.

We consider solving a regression problem over a wireless network using a real-world dataset. To this end, we extract a binary dataset from MNIST (hand-written digits) by keeping only samples of digits 0 and 1 and then setting their labels to -1 and +1 , respectively. We then randomly split the resulting dataset of 12600 samples among $M$ worker nodes, each having $\left\{\left(\boldsymbol{x}_{i j}, y_{i j}\right)\right\}$, where $\boldsymbol{x}_{i j} \in \mathbb{R}^{784}$ is a vectorized image at node $j \in[M]$ with corresponding digit label $y_{i j} \in\{-1,+1\}$. Without loss of generality, we consider a convex loss function

$$
f(\boldsymbol{w})=\frac{1}{M} \sum_{j \in[M]} \frac{1}{N_{j}} \sum_{i \in\left[N_{j}\right]} \log \left(1+e^{-\boldsymbol{w}^{T} \boldsymbol{x}_{i j} y_{i j}}\right)
$$

where we consider that each worker node $j \in[M]$ has the same number of samples, namely $N_{j}=N_{l}, \forall j, l \in[M]$.

In our experiments, we consider heavy $\left(p_{b}=0.2\right)$ and light $\left(p_{b}=0.02\right)$ traffics, and dense $(M=10)$ and sparse $(M=5)$ networks. We run the network for $10^{3}$ times and define $c_{k}$ as the latency (in seconds) required to receive the gradient vectors by master node and run iteration $k$. This latency corresponds to receiving all the gradients batch update and $T_{s}$ time slots for mini-batch update. We also set $\beta=0.5$.

\section{A. Batch Updates}

Here, we evaluate the impact of batch gradient updates on the performance of networked AI solving (1). Then, we use our Algorithms 3 and 2 to solve (6) as means to find costefficient solutions for (1).

Fig. 2 illustrates $f, \sum_{k} c_{k}$, and $G$ against $\bar{k}$. Fig. 2(a) confirms Proposition 2 and shows that our simple casual algorithm can almost recover the optimal stopping criteria, obtained by the non-causal setting. Unless otherwise mentioned, the following figures, including Figs. 2(b) and 2(c), show the results for the causal setting. Fig. 2(b) illustrates the cost of running distributed gradient descent for various background traffics and network densities. Adding more worker nodes,

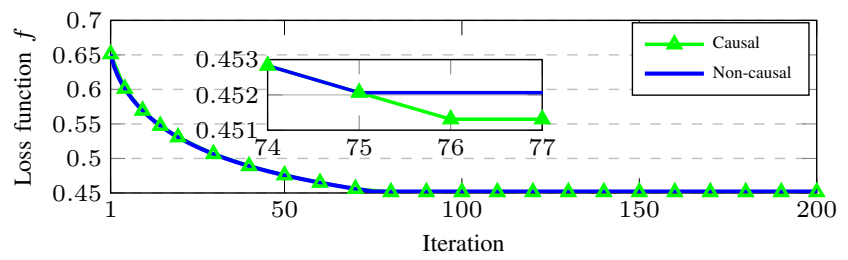

(a)

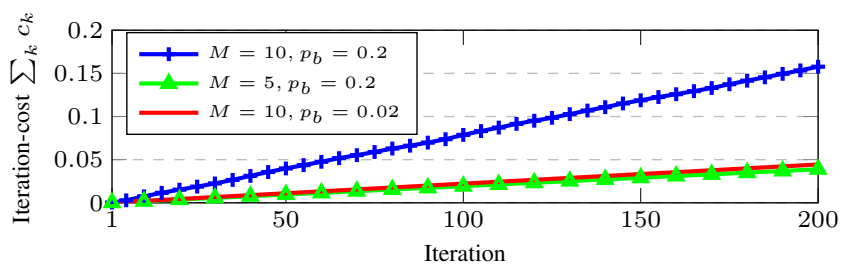

(b)

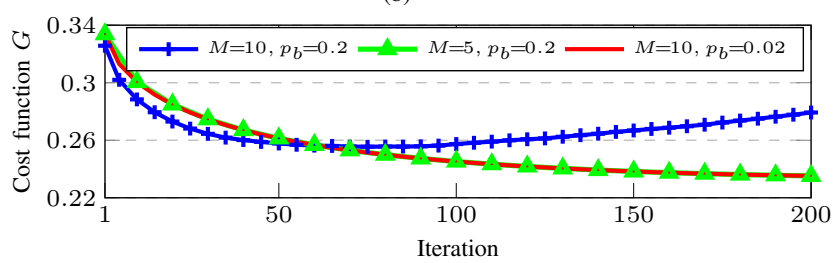

(c)

Fig. 2: Illustration of the batch update Algorithm 2.

while reducing the computational loads of every worker node, may lead to a substantial penalty in the latency cost for running every iteration due to higher network congestion. Moreover, heavier background traffics, if not handled properly by the medium access control layer, would increase the latency of running every iteration.

\section{B. Mini-batch Update}

In the mini-batch update, we wait for $T_{s}$ time slots. The master node then updates the parameter using the gradients it received during this period. We also set $n_{t}=15$, computed by cross-validation, to reduce the effect of noise in the gradient estimates and stopping criteria.

Fig. 3 characterizes the number or received gradients and mean queue length of the worker nodes against iteration number $\bar{k}$. From this figure, network congestion $\left(M=10, p_{b}=\right.$ 0.2 ) drastically increases the queue length of the worker nodes and therefore sending $\nabla_{k}^{j}$ for higher $k$ may involve a higher latency. Consequently, it is likely that after some iterations, the huge backlogged queue would not allow for getting enough gradient vectors during $T_{s}$ slots. In that case, although we are paying the cost of running iterations (exchanging parameters and gradients), there is almost no progress in the optimization, and the gradient descent can converge only to a ball around the minimizer $\boldsymbol{w}^{\star}$, whose radius is determined by the missing gradient vectors [4]. This can be addressed by increasing $T_{s}$ (to collect more gradients) at the expense of higher per-iteration latency. Such extra latency may not be necessary for light background traffics, e.g., $\left(M=5, p_{b}=0.2\right)$. These results highlight the importance of the co-design of machine learning algorithms and wireless networking protocol. 


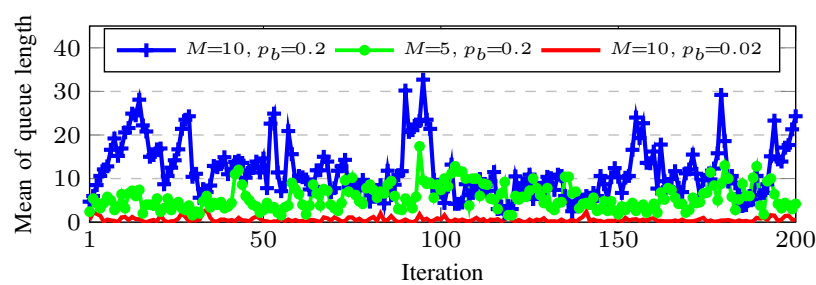

(a)

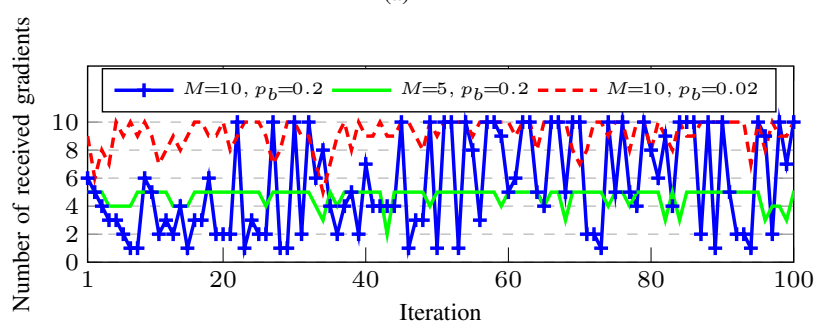

(b)

Fig. 3: Illustration of the mini-batch update Algorithm 3.

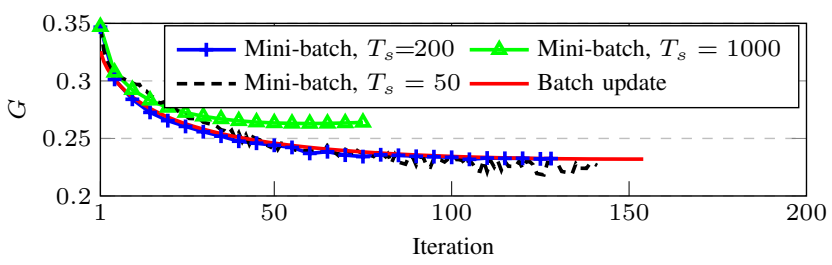

(a) Cost function.

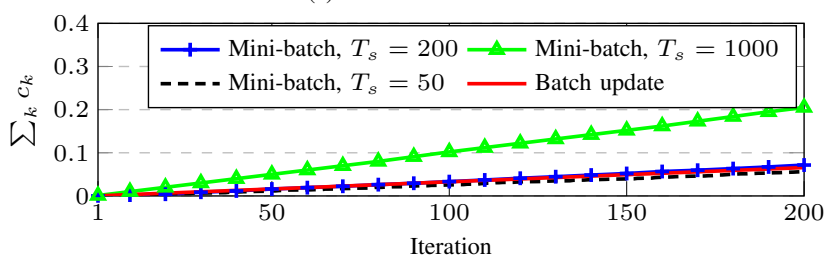

(b) Iteration-cost function.

Fig. 4: Comparison between batch and mini-batch updates of Algorithm 2 and 3 , respectively, with $M=10$ and $p_{b}=0.1$.

Fig. 4 compares the objective function $G(\bar{k})$ and the iteration cost $\sum_{k=1}^{\bar{k}} c_{k}$ of batch and mini-batch updates for $M=10$ and $p_{b}=0.1$. This figure highlights the importance of designing communication protocol. An improper choice of $T_{s}$ may lead to a very bad solution, both in terms of loss function $f$ and cost $\sum c_{k}$. However, with a proper design, the mini-batch approach can outperform batch updates. The minibatch update allows for running more less-expensive iterations than the batch update counterpart. As a result, we have a faster convergence to a vicinity of the minimizer $\boldsymbol{w}^{\star}$ until the high iteration-cost prohibits running more iterations and getting closer to $\boldsymbol{w}^{\star}$. Moreover, the mini-batch approach is robust to the straggler worker nodes without any need for adding redundancy to the optimization problem as done in [18].

\section{CONClusion}

In this paper, we proposed a framework to design cost-aware distributed optimization for ML over Networks (MLoN). We characterized the cost of running iterations of a generic gradient-based distributed solution algorithms over a shared wireless channel, regulated by a slotted-ALOHA protocol. We characterized the end-to-end latency, as the iteration-cost function, and showed that increasing the background traffic may prohibit the application of MLoN. We optimized the iteration-termination criteria to minimize the trade-off between achievable objective value and the overall cost of running the solver. We concluded that a co-design of distributed optimization algorithms and communication protocols are essential for the success of MLoNs.

\section{REFERENCES}

[1] M. I. Jordan and T. M. Mitchell, "Machine learning: Trends, perspectives, and prospects," Science, vol. 349, no. 6245, pp. 255-260, 2015.

[2] H. Li, K. Ota, and M. Dong, "Learning iot in edge: Deep learning for the internet of things with edge computing," IEEE Network, vol. 32, no. 1, pp. 96-101, 2018.

[3] J. Park, S. Samarakoon, M. Bennis, and M. Debbah, "Wireless network intelligence at the edge," CoRR, vol. abs/1812.02858, 2018. [Online]. Available: http://arxiv.org/abs/1812.02858

[4] L. Bottou, F. Curtis, and J. Nocedal, "Optimization methods for largescale machine learning," SIAM Review, vol. 60, no. 2, pp. 223-311, 2018.

[5] J. Konečnỳ, H. B. McMahan, F. X. Yu, P. Richtárik, A. T. Suresh, and D. Bacon, "Federated learning: Strategies for improving communication efficiency," arXiv preprint arXiv:1610.05492, 2016.

[6] R. Johnson and T. Zhang, "Accelerating stochastic gradient descent using predictive variance reduction," in Advances in neural information processing systems, 2013, pp. 315-323.

[7] W. Chen, Y. Wang, and Y. Yuan, "Combinatorial multi-armed bandit: General framework and applications," in International Conference on Machine Learning, 2013, pp. 151-159.

[8] J. Bernstein, Y.-X. Wang, K. Azizzadenesheli, and A. Anandkumar, "signSGD: compressed optimisation for non-convex problems," arXiv preprint arXiv:1802.04434, 2018.

[9] S. Magnússon, H. S. Ghadikolaei, and N. Li, "On maintaining linear convergence of distributed learning and optimization under limited communication," arXiv preprint arXiv:1902.11163, 2019.

[10] H. Yu, S. Yang, and S. Zhu, "Parallel restarted SGD for non-convex optimization with faster convergence and less communication," arXiv preprint arXiv:1807.06629, 2018.

[11] M. Ivanov, F. Brännström, A. Graell i Amat, and P. Popovski, "Broadcast coded slotted aloha: A finite frame length analysis," IEEE Transactions on Communications, vol. 65, no. 2, pp. 651-662, Feb 2017.

[12] H. Shokri-Ghadikolaei and C. Fischione, "The transitional behavior of interference in millimeter wave networks and its impact on medium access control," IEEE Trans. Commun., vol. 62, no. 2, pp. 723-740, Feb. 2016.

[13] Y. Xu and W. Yin, "A block coordinate descent method for regularized multiconvex optimization with applications to nonnegative tensor factorization and completion," SIAM Journal on Imaging Sciences, vol. 6 , no. 3, pp. 1758-1789, 2013.

[14] Y. Yang and T. Yum, "Delay distributions of slotted ALOHA and CSMA," IEEE Trans. Commun., vol. 51, no. 11, pp. 1846-1857, Nov. 2003.

[15] F. S. Samani, H. Zhang, and R. Stadler, "Efficient learning on highdimensional operational data," in 15th IEEE International Conference on Network and Service Management (CNSM), 2019.

[16] Ü. Yüceer, "Discrete convexity: convexity for functions defined on discrete spaces," Discrete Applied Mathematics, vol. 119, no. 3, pp. 297-304, 2002.

[17] K. Murota, Discrete convex analysis. SIAM, 2003.

[18] C. Karakus, Y. Sun, S. N. Diggavi, and W. Yin, "Redundancy techniques for straggler mitigation in distributed optimization and learning." Journal of Machine Learning Research, vol. 20, no. 72, pp. 1-47, 2019. 